\title{
Reflexiones y Perspectivas sobre los Usos de las Redes Sociales en Educación. Un Estudio de Caso en Quito-Ecuador
}

\author{
Andrés Hermann-Acosta ${ }^{1 *}$, Diego E. Apolo ${ }^{2,3}$ y Milton Molano-Camargo ${ }^{4}$ \\ (1) Universidad Tecnológica Indoamérica. Av. Machala y Sabanilla. EC170103 Quito-Ecuador. \\ (2) Universidad Nacional de Educación- UNAE, EC030203 Antigua Panamericana. Javier Loyola-Ecuador. \\ (3) Universidad de Las Américas, EC170125 Quito, Ecuador. \\ (4) Universidad de La Salle, Cra. 5A No 59 A 54 Bogotá-Colombia \\ (e-mail: andreshermann@uti.edu.ec; diego.apolo@unae.edu.ec; mmolano@lasalle.edu.co)
}

* Autor a quien debe ser dirigida la correspondencia

Recibido Jun. 4, 2018; Aceptado Sep. 24, 2018; Versión final Oct. 10, 2018, Publicado Feb. 2019

\begin{abstract}
Resumen
Este estudio se enfocó en determinar cómo el uso de los recursos de la web 2.0 como las redes sociales está permitiendo la construcción de aprendizajes, en contextos educativos no formales mediados por el uso de tecnologías digitales. Para la obtención de los datos de la investigación se aplicó un enfoque mixto de alcance descriptivo-exploratorio, el mismo que articuló una revisión de fuentes secundarias de alto impacto, así como la aplicación de un estudio de caso, basado en encuestas realizadas a estudiantes de educación media en Quito-Ecuador. Se estableció que un $80 \%$ de los jóvenes usuarios de medios han utilizado las redes sociales con fines educativos, aspecto que ha posibilitado llegar a la conclusión que el uso de tecnologías digitales no sólo está contribuyendo a fomentar aspectos como la comunicación y el entretenimiento, sino también incorporar aprendizajes invisibles a sus procesos cognitivos.
\end{abstract}

Palabras clave: redes sociales jóvenes; educación no formal; educación informal; aprendizajes invisibles.

\section{Reflections and Perspectives on the Uses of Social Networks in Education. A Case Study in Quito-Ecuador}

\begin{abstract}
This research focused on determining how the use of web 2.0 resources such as social networks is allowing the construction of learning processes, in non-formal educational contexts mediated by the use of digital technologies. To obtain the data a mixed research method was applied, including a review of high-impact secondary sources, as well as the application of a case study, based on surveys of high school students in Quito-Ecuador. It was established that $80 \%$ of young media users have used social networks for educational purposes, an aspect that has made it possible to conclude that the use of digital technologies is not only helping to promote aspects such as communication and entertainment, but also incorporate invisible learning to their cognitive processes.
\end{abstract}

Keywords: social networks; youth; non-formal education; informal education; invisible learning. 


\section{INTRODUCCIÓN}

El presente estudio, parte del análisis de los resultados presentados por Hermann (2015) quien propone la reflexión de la tecnología en el campo educativo desde una visión sociocrítica con énfasis en la construcción de conocimientos y aprendizajes invisibles que pongan en debate visiones que enfatizan el abordaje de la escuela como reproductora de conocimientos que responde a un proyecto de modernidad y colonialidad del poder.Para ello es relevante recurrir a aportes de Althusser (2003) quien demuestra como las instituciones en su constitución debieron adscribirse a un proyecto de Estado-nación hacia una lógica neocolonial que no se encarga de formar a los sujetos para mejorar sus condiciones sociales; sino, más bien su propuesta se ha enfocado históricamente en generar la división de clases, ideologizar y someter socialmente por parte de la clase privilegiada hacia los grupos subalternos. La escuela, la iglesia y la familia funcionan como aparatos ideológicos del estado que se han encargado de mantener el orden social mediante la homogeneización de ciudadanos quienes son juzgados por su atención a la dualidad mentecuerpo desplazando aspectos culturales que requieren ser observados (García-Carrasco, 2017). A partir de estas perspectivas, se puede evidenciar que la función que ha venido ejerciendo la escuela tradicional ha desplazado la construcción de sujetos críticos y creativos, y más bien ha reforzado la configuración de individuos que se adecúen a un enfoque economicista donde prime un currículo cerrado y estandarizado, que no cuestione; sino que cumpla. Lo cual no dialoga con diferentes propuestas que enuncian la relevancia de un acompañamiento que emplee abordajes como la didáctica invertida y aprendizajes en línea como espacios abiertos de apropiación de conocimientos y participación democrática (Kaun, Kyriakidou y Uldam, 2016; Fadol, Aldamen y Saadullah, 2018).

Retomando aportes de Althusser (2003) en diálogo con propuestas Lytoard (2000), se afirma la idea de que la educación formal posee una intencionalidad fundacional dirigida, donde el esquema no es educar para responder las necesidades formativas de los educandos; sino naturalizar el proyecto de la colonialidad del poder. Es por esta razón que este estudio pretende presentar aportes que permitan valorar, rescatar y resaltar los distintos aprendizajes desde la educación no formal e informal que realizan los actores del proceso educativo como lo demuestran diferentes estudios (Morillo, Sebastián y Casado, 2011; Schei y Nerbø, 2015; Smith, 2016). Para lograr aterrizar la fundamentación de la crítica a la escuela como espacio de homogenización del pensamiento y la emergencia de tecnologías digitales como las redes sociales, se ha tomado como estudio de caso dos instituciones educativas particulares de Quito-Ecuador, el "Colegio Particular Antares" y el "Liceo del valle", a partir de la aplicación de 396 encuestas a estudiantes de entre 12 y 18 años, los cuales permitieron evidenciar que el uso de las redes sociales, no sólo están contribuyendo a generar procesos comunicacionales y de entretenimiento, sino que se logra configurar una educación abierta, flexible y descentralizada.

En la actualidad, el uso de las herramientas tecnológicas de las plataformas de web 2.0, como blogs, wikis, redes sociales, entre otras herramientas, poseen un gran impacto en la formación de los jóvenes fomentado los usos sociales (Caldevilla, 2010; Akcaoglu y Bowman, 2016; Pedro, Barbosa y Santos, 2018), es así como también las redes sociales se han conformado como nuevos espacios de interacción y por ende de aprendizaje abierto constituyendo una ruptura de los procesos de dominación y legitimación de los discursos, meta-relatos y saberes cientificistas impulsados por las elites intelectuales. Antes de dar paso a la reflexión de lo que implican las nuevas formas de educación en la praxis educativa en la era digital, es de gran importancia definir la tipología de cada uno de los ámbitos de intervención de las modalidades de estudio, así en la educación formal se entiende como un esquema de transferencia de contenidos que se adscribe a una lógica institucionalizada, definida por un currículo regulada por la política educativa (Hermann, 2015).

Con la eclosión de las Tecnologías de la Información y la Comunicación en la década de los 90, varios ámbitos sociales replantearon sus prácticas, el caso del sector educativo no quedó exento. El uso de espacios como los entornos digitales y las redes sociales han permitido pensar espacios formativos, que no sólo se sostienen sobre la base del logro de certificaciones académicas y el consumo de contenidos de estudio; sino, más bien de aspectos vinculados con responder necesidades e intereses de los educandos a partir de comunidades del aprendizaje que se auto-regulan. En tal sentido, no se pretende sostener que la educación formal tendrá que ser remplazada por la educación no formal o informal; sino; más bien que se podría alimentar y tomar de estas modalidades de estudios emergentes estrategias que permitan entender a la educación como un espacio flexible, abierto, descentralizado, colaborativo y co-creado desde espacios no formales e informales que se construyan a partir de las ideas, experiencias y conocimientos que se dan a partir de la cotidianeidad, tal como se expresa en la Tabla 1. El elemento sustantivo que sostiene este estudio, parte de la premisa que es posible dar un giro al esquema educativo tradicional, que en gran parte de los casos está adscrito a una modalidad de estudios presencial y que reproduce los elementos del tipo de educación formal, donde el valor primordial reside en la adquisición de competencias que aportan al desarrollo de mano de obra cualificada, y no a la formación del educando desde sus intereses y dar respuesta a la resolución de problemas de sus contextos personales y sociales. 
Tabla 1: Espacios no formales e informales. (Adaptada de Hermann, 2015)

"En lo que respecta a la educación no formal, este tipo de estudios, se caracteriza porque no responde a la organización del sistema educativo regular y escolarizado, esta variante formativa se lleva a cabo en centros de actualización y capacitación, que no están adscritas a las instituciones educativas reguladas por la política educativa" (p.23)
"En lo que corresponde a la educación informal, se entiende como aquella acción formativa capaz de generar adquisición de conocimientos y aprendizajes de manera involuntaria y no consciente. A este tipo de actuaciones educativas corresponde gran parte de las experiencias que logramos día a día desde el interrelacionamiento social y de la cotidianeidad" (p. 24)

De esta forma, se establece sin caer en generalizaciones que todos los jóvenes son nativos digitales, que por lo menos han tenido acercamientos a la tecnología en diferentes niveles y por tanto es relevante conocer sus experiencias y usos. Partiendo de ello observar la manera en cómo han adquirido sus conocimientos vinculando las TIC y han logrado incorporar un currículo oculto, donde desde aportes de Cobo y Moravec, (2011), se ha logrado consolidar los aprendizajes invisibles, que tienen relación con la adquisición de nuevos saberes sociales que se dan a partir de la interacción en red y sobre todo y en diálogo con lo anterior, diferentes autores (Nehls y Livengood, 2017; Martínez-Argüello, Hinojo-Lucena y Díaz, 2018) consideran que es relevante desde los sistemas de educación tomar en cuenta estas apropiaciones que se desarrollan en los espacios no formales e informales con el fin de responder a los contextos de los actores del proceso educativo se enfrentan en el cotidiano.

\section{EL APRENDIZAJE ABIERTO EN ENTORNOS DIGITALES}

Durante el primer decenio del siglo XXI, los Entornos Virtuales de Aprendizaje (LMS) aportaron a la educación basada en herramientas tecnológicas escolásticas y cerradas; ya que, lo común era que se reproduzcan las prácticas de la educación presencial, tradicional y formal en las aulas virtuales, respondiendo a un currículo basado en contenidos de estudio y no en el logro de los resultados de aprendizaje. Esta perspectiva fue el eje de abordaje que un primer intento de emplear plataformas digitales en ámbitos educativos. A partir de ello existieron aportes que proponían reflexionar sobre los diferentes tipos de escenarios y recursos para el aprendizaje en la era digital. En este contexto Santamaría (2012), propone entender a los escenarios educativos abiertos como nuevos ecosistemas de interacción donde el énfasis se encuentra en el desarrollo y estructura de prácticas educativas abiertas, mismas que vinculan estrategias de apropiación del conocimiento desde los espacios cotidianos con fines educativos. Es así que estas prácticas educativas abiertas deben hacer uso de diferentes herramientas como Sistemas Gestores de Contenido (CMS), Entornos Personales de Aprendizaje (PLE), Recursos de la Web 2.0 como los blogs, wikis, redes sociales, Cursos Online Masivos y Abiertos (MOOC); ya que, permiten observar a las plataformas digitales y redes sociales como espacios que superan la visión de una educación enciclopedista, tecnocrática y unidireccional, hacia un tipo de educación flexible, abierta y descentralizada donde los actores del proceso educativo juegan papel relevante como prosumidores y eduprosumidores pasando de únicamente consumir contendidos a producirlos y compartirlos en este caso con fines educativos. (Toffler, 1980; Apolo et al. 2017). Con la idea de fortalecer el concepto de educación en espacios abiertos, se presentan, en la figura 1, algunas características de este nuevo esquema educativo:

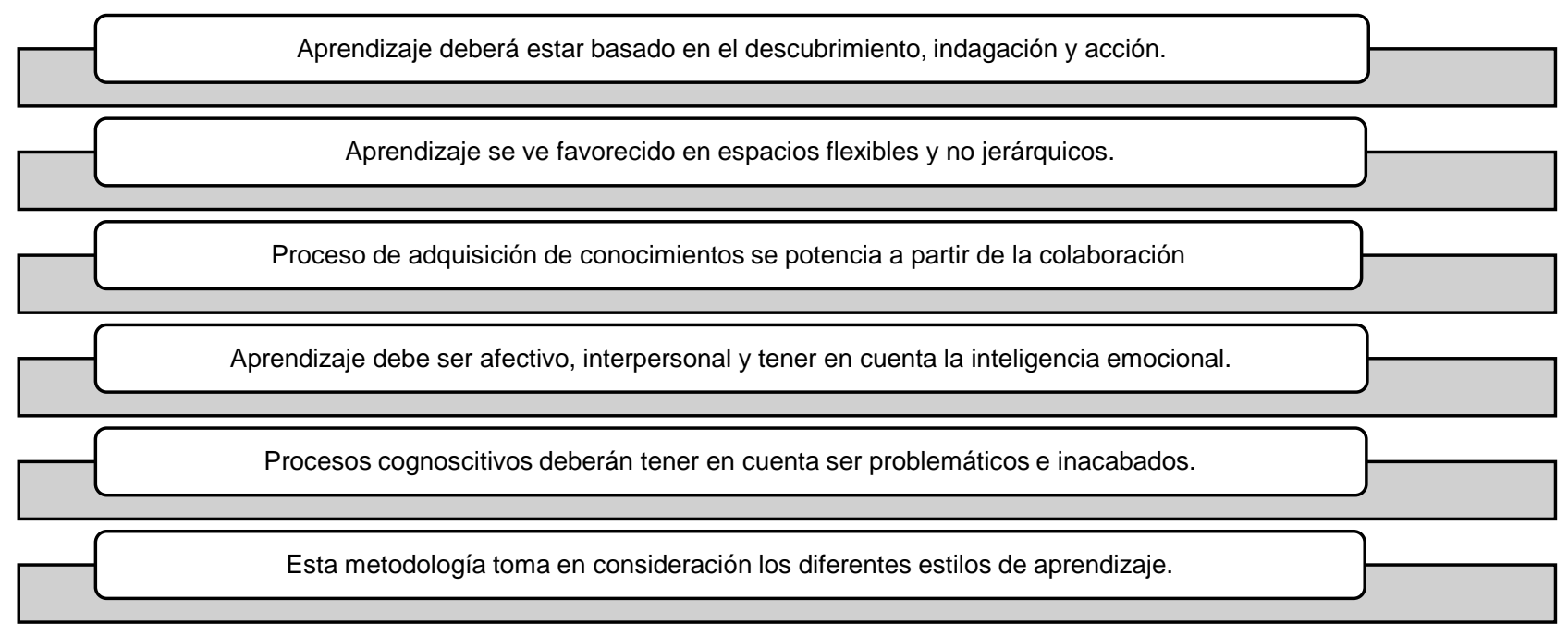

Fig. 1: Características de la educación en espacios abiertos. (Adaptada de Santamaría 2012) 
Una de las motivaciones por la cual el presente trabajo se enfocó en desarrollar un estudio de caso; es que, no se puede negar que las prácticas educativas abiertas se ven favorecidas desde las redes sociales; ya que, estas herramientas descentralizan y se articulan bajo la lógica de los principios antes expuestos, valorando espacios de educación informal, donde la adquisición de nuevos conocimientos se dan a partir del aprendizaje por descubrimiento, práctico lo cual fomenta la inteligencia interpersonal basada en las emociones y sentimientos que brindan estos espacios digitales (Colás, González y De Pablos, 2013; Abdelraheem y Ahmed, 2018). El planteamiento que trae la experiencia de generar educación flexible y abierta a partir de las redes sociales se orienta con el fin de impulsar la configuración de un tipo de educación interactiva, dialógica basada en el uso de las TIC y anclada con esquemas de formación de la educación no formal e informal.

Es así, donde las redes sociales a diferencia de otros espacios y herramientas centradas en la unidireccionalidad de sus contendidos, ésta fomenta el trabajo en equipo, la investigación y el compartir experiencias desde usuarios a usuarios. Por ende, es relevante trabajar por una educación multidireccional, dialógica e interactiva, donde las audiencias puedan pasar a ser de receptoras de datos e información hacia creadoras, colaboradoras y cooperantes en la construcción de contenidos. Para ello es necesario reconocer los conocimientos y competencias que adquieren los jóvenes fuera de las cuatro paredes del aula como en muchas ocasiones se concibe a la escuela y permitiendo aportar hacia un abordaje que fomente el vínculo socio humanístico entre tecnología y educación. La tabla 2 presenta una síntesis de algunas de las transformaciones que el ciberespacio genera y que impactan las subjetividades de los estudiantes, sus formas de aprender, el papel de las mediaciones e incluso las posibilidades del acompañamiento.

Tabla 2: Características de la educación en espacios abiertos. (Adaptada de Rodríguez 2013).

\begin{tabular}{|c|c|c|}
\hline Espacios de Transformaciones & Explicación & Impacto pedagógico \\
\hline $\begin{array}{l}\text { En la representación, captación } \\
\text { y manejo de la información }\end{array}$ & $\begin{array}{l}\text { Disolución de la dicotomía } \\
\text { análogo-digital. Se potencian } \\
\text { los dispositivos de } \\
\text { información. Se impone un } \\
\text { imperativo de eficiencia y } \\
\text { alcance de la comunicación. }\end{array}$ & $\begin{array}{l}\text {-Ambientes digitales de aprendizaje. } \\
\text {-Acceso a diferentes fuentes de } \\
\text { información y necesidad de criterios } \\
\text { de calidad para elegir } \\
\text {-Incentivo al autoaprendizaje. } \\
\text { Estudiantes y profesores como } \\
\text { Prosumer. }\end{array}$ \\
\hline En la corporalidad & $\begin{array}{l}\text { - "Protesticidad" prótesis } \\
\text { tecnológicas } \\
\text { - Multisensorialidad. } \\
\text { Exposición a múltiples } \\
\text { modalidades comunicativas. }\end{array}$ & $\begin{array}{l}\text {-Incorporación creciente de artefactos } \\
\text { a la cotidianidad del aula } \\
\text {-Realidad virtual, realidad aumentada, } \\
\text { simuladores incorporados al } \\
\text { aprendizaje. Otra forma de someter a } \\
\text { prueba las hipótesis, ajustar modelos } \\
\text { teóricos, aprovechar el error como } \\
\text { fuente de crecimiento. } \\
\text { - Nuevas formas de lectura y } \\
\text { escritura. Fenómeno youtuber. }\end{array}$ \\
\hline $\begin{array}{l}\text { En la funcionalidad de la } \\
\text { infraestructura infotecnológica }\end{array}$ & $\begin{array}{l}\text { Reconfiguración neurológica } \\
\text { del cerebro }\end{array}$ & $\begin{array}{l}\text {-Necesidad de investigación en el } \\
\text { campo. } \\
\text { - La pregunta por los nuevos saberes } \\
\text { (Edgar Morin) }\end{array}$ \\
\hline $\begin{array}{l}\text { En las concepciones o } \\
\text { percepción de las categorías de } \\
\text { tiempo y espacio }\end{array}$ & $\begin{array}{l}\text { "Distalidad" Naturalización de } \\
\text { los contactos a distancia. } \\
\text { "Reticularidad" pensamiento } \\
\text { en redes, no lineal. } \\
\text { Nueva movilidad sin } \\
\text { desplazarse } \\
\text { "Instantaneidad" moverse en } \\
\text { tiempo real } \\
\text { independientemente del lugar } \\
\text { "Ubicuidad" Conexión } \\
\text { permanente. }\end{array}$ & $\begin{array}{l}\text { - Incorporación de trabajos } \\
\text { colaborativos y cooperativos } \\
\text { mediados por redes. } \\
\text { - Mejores posibilidades para el trabajo } \\
\text { independiente del estudiante. } \\
\text { - Nuevo rol del profesor en relación } \\
\text { con las mediaciones y el acceso al } \\
\text { aprendizaje. Desplazamientos en el } \\
\text { poder. } \\
\text { - Empoderamiento de los procesos e- } \\
\text { learning }\end{array}$ \\
\hline
\end{tabular}




\section{MATERIALES Y MÉTODOS}

Para este estudio se empleó un enfoque de investigación mixto, desde los aportes de diferentes autores (Parra, Gordo y D’Antonio, 2014; Velásquez, Carrión y Yaguache 2016), mediante el uso del enfoque cualitativo con base en la revisión bibliográfica y cuantitativa referente a la aplicación de encuestas a jóvenes pertenecientes a dos instituciones educativas privadas ubicadas en Quito-Ecuador. En cuanto a la caracterización etaria los informantes se encuentran en edades entre los 12 y 18 años, varones y mujeres que asisten a los tres últimos niveles académicos del Bachillerato General Unificado del país. Para la determinación del número de informantes se tomó la decisión de realizar las encuestas al universo total de 396 estudiantes pertenecientes a los dos colegios donde se aplicó este estudio desde la siguiente ruta, según la figura 2, de la metodología de investigación.

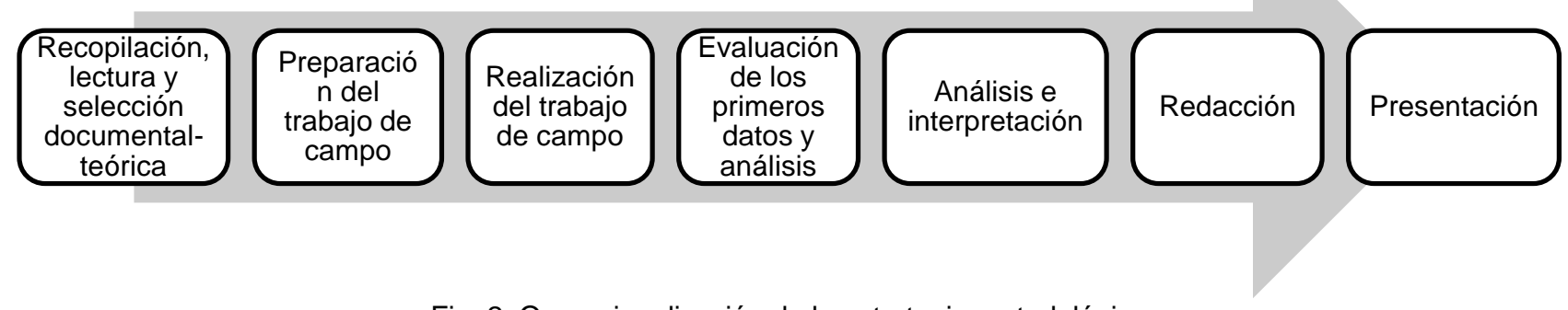

Fig. 2: Operacionalización de la estrategia metodológica

\section{RESULTADOS Y DISCUSIÓN}

En cuanto a género se recogió la información de varones en un $51 \%$ y mujeres $48 \%, 1 \%$ de informantes no respondieron esta pregunta. Se realizó en primer lugar una descripción desde los resultados referentes al acceso y conexiones, para posteriormente continuar con aproximaciones vinculadas al uso de las redes sociales y educación para terminar con aspectos relacionados con la percepción que tienen los jóvenes sobre el uso de las tecnologías en la educación. En este punto es importante destacar que esta investigación vio como favorable el potenciar el proceso de educación sustentado en herramientas como las redes sociales por dos factores, el primero que tiene que ver con condiciones de infraestructura tecnológica, ya que en el Ecuador según estudios del MINTEL (2015) el 52.45\% dispone de un computador en el hogar y el resto de la población que responde al $47.58 \%$ si bien es cierto no cuenta con condiciones de conectividad, al menos tiene accesibilidad a la red internet a partir de lugares comunes donde se cuenta con este servicio. El segundo factor que favorece el re-plantear el esquema educativo a partir de las redes sociales, es que estas herramientas son bien manejados por los estudiantes jóvenes usuarios de medios. Lo que está de acuerdo con la investigación de Medina, Lagunes y Torres (2018) quienes concluyen que la percepción de los estudiantes acerca del uso de recursos tecnológicos como las redes sociales es que es interesante, divertido, fácil y concreto, y que investigar sobre estas opiniones, según los estudios sobre el estado de la investigación en estos aspectos es importante por la combinación de componentes cognitivos y emocionales que tienen relación con la predisposición al aprendizaje.

Por otro lado, se pudo establecer que el $99 \%$ de informantes cuenta con un computador en sus casas, lo cual permite dentro de otras actividades emplearlo para entretenimiento y educación. Dentro de los informantes que cuentan con computador en sus casas, el $52 \%$ menciona que cuenta con un computador en sus habitaciones esto hace referencia hacia el acceso a dispositivos en espacios privados dialogando con los porcentajes de penetración de dispositivos en hogares donde se evidencia un amplio crecimiento del acceso a computador móvil Ecuador (INEC, 2016), como puede verse en la figura 3.

En cuanto al uso de dispositivos como celulares inteligentes el $90 \%$ de informantes los poseen y dentro de ellos el $47 \%$ paga un plan de datos. Bajo estos resultados se estableció mediante meta-análisis que las edades de quienes respondían poseerlo oscila entre los 17 y 18 años y son quienes están por ingresar a instituciones de educación superior. Se pudo determinar también que los tres principales lugares desde donde acceden los jóvenes son: con un $40 \%$ desde sus hogares, $27 \%$ desde el colegio y el $11 \%$ desde espacios públicos. Este dato es relevante; ya que, permite identificar como la penetración de internet en los últimos años ha permitido su alcance hasta los hogares. Es relevante mencionar ello, tomando en cuenta que, en los inicios de la penetración de internet al país, el acceso era casi nulo desde los hogares por su costo y cobertura. De igual manera, este dato dialoga con las horas que destinan al uso de internet donde se establece que el $32 \%$ de informantes se conecta entre 3 y 4 horas, el $24 \%$ entre 5 y 6 horas y el $23 \%$ entre 1 y 2 horas. 
A la pregunta si son usuarios de alguna red social el $98 \%$ de jóvenes responde afirmativamente, de igual manera se determina que el $64 \%$ de estos se conectan entre 1 y 4 horas diarias a este tipo de plataformas. La tendencia muestra que las tres principales redes sociales son: Facebook, Whatsapp e Instagram. En cuanto a las principales actividades que realizan cuando se conectan a redes sociales destacan: comunicación con un $21 \%$, entretenimiento con $18 \%$ y videojuegos con el $10 \%$. Con respecto al uso de redes sociales con fines educativos el $80 \%$ mencionan que, sí las usan en temas escolares, de ellos el $29 \%$ consideran que éstas han contribuido entre rangos de muy alto y alto a sus procesos formativos. Esto concuerda con los resultados del estudio de Martínez, Hinojo y Aznar (2018) quienes muestran en su investigación sobre el uso de las redes sociales con fines educativos en la enseñanza de la química, que el $21,7 \%$ de estudiantes declararon haberlas usado frecuentemente.

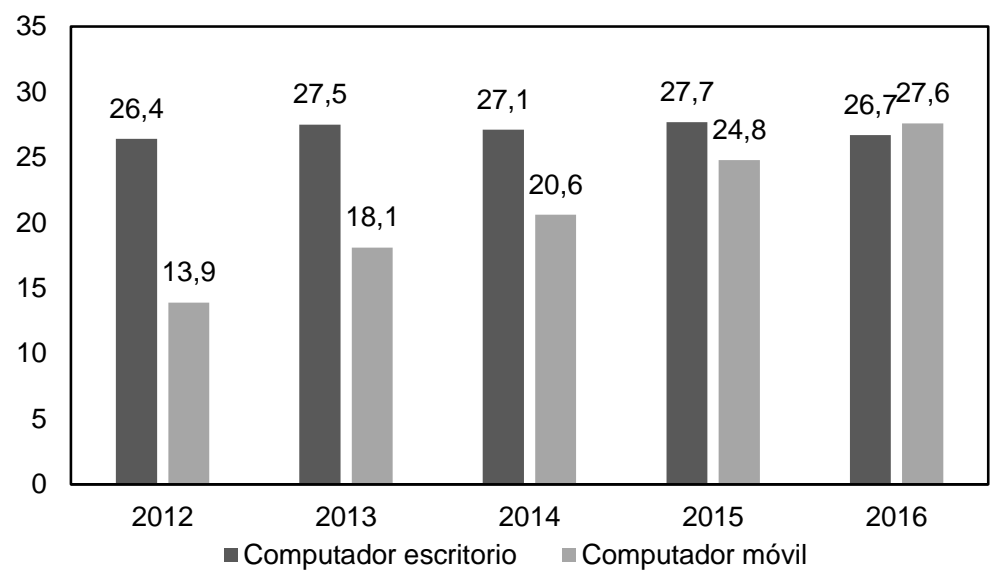

Fig. 3: Hogares con computador de escritorio y móvil en casa en Ecuador (Adaptado de INEC, 2016)

En relación a la consulta sobre el lugar que consideran los jóvenes usuarios de medios aprenden de mejor manera, consta en primer lugar los centros educativos con un $30 \%$, seguido por espacios familiares con $22 \%$ y con el $21 \%$ desde entornos tecnológicos mediados por la red internet. Ello muestra como existe aún predominancia de los espacios formales, seguidos por los espacios no formales como es el núcleo familiar y en tercer lugar aprendizajes desde espacios informales mediados por el uso de las TIC. En lo referente al uso de redes sociales, el $93 \%$ de los padres de familia cuentan con perfiles en redes sociales, este dato permite identificar que que cada vez más padres están inmersos en el manejo de estos entornos tecnológicos y que no es necesario que desarrollen un uso fluido, sino que al menos ya tienen presencia en estos medios virtuales, lo que de alguna forma ayuda para que los jóvenes puedan tener orientaciones con criterios por parte de los adultos. Esto tiene una alta relación con estudios en diversos contextos geográficos (Siibak, Forsman y Hernwall, 2012; Park, 2016; Kristianto,2017; Lubua, Semlambo, y Pretorius 2017) que confirma muchos de los efectos sobre la actitud de los niños y jóvenes hacia las redes sociales, a partir de la influencia de los padres, entre otros aspectos, como la interacción con los pares, la facilidad de uso, el nivel de acceso, la experiencia de usuario y la consciencia de riesgo.

En cuanto a mantener un diálogo sobre el uso de redes sociales con los docentes, se ha logrado identificar que el $81 \%$ si lo ha hecho uso de estas herramientas para generar aportes de orientaciones en la indagación de información. De igual manera el $67 \%$ de estudiantes mencionan haber recibido algún tipo de capacitación sobre el uso adecuado de redes sociales por parte de su centro educativo. Es así como surge la relevancia que las instituciones fomenten un uso significativo de las tecnologías en la educación valorando el uso que hacen los jóvenes de las redes sociales y por ende a los nuevos entornos de socialización permitirán aprovechar sus apropiaciones para fomentar estrategias que potencien aprendizaje autónomo, el trabajo en equipo y la conexión de redes académicas de manera global.

A este respecto distintas investigaciones (Vásquez y Cabero 2015; Alcalá, Ortiz y Flores 2015; Martínez, Hinojo y Aznar 2015; Rodríguez, Haya y Fernández, 2015) evidencian los beneficios y el desarrollo potencial del uso de redes sociales en el aprendizaje ya que incrementan la comunicación e interacción, propician la construcción colectiva del conocimiento a través de procesos de aprendizaje colaborativos y promueven el desarrollo de competencias y estrategias cognitivas y metacognitivas complejas. Sin embargo, para que esto sea posible el docente debe hacer un proceso consciente y profesional que le permita comprender el sentido del uso de las redes sociales con propósitos de aprendizaje, más allá del simple compartir información y de posiciones ingenuas que asumen que su mera utilización puede apoyar una perspectiva constructivista cuando en realidad ello va a depender de la estrategia metodológica que se aplique sobre las mismas. Además, es relevante estar convencido del valor pedagógico y didáctico y comprometerse en el 
empoderamiento de los estudiantes para un uso más autónomo y menos caprichoso de la red. Todo esto plantea nuevos desafíos a la comunidad académica desde una perspectiva epistemológica y metodológica dado que estos entornos están mediados por condiciones tales como la inmediatez, el tamaño reducido de los contenidos que se moviliza o la necesidad de vínculos personales en las relaciones entre los usuarios. Por lo que es necesario desarrollar nuevas estrategias para el desarrollo de investigaciones que respondan a estos desafíos y que permitan la reconstrucción de una imagen compleja de la interacción que ocurre en estos entornos (Rodríguez, Haya y Fernández, 2015).

En cuanto a la percepción de las tecnologías y su aporte en educación se pudo establecer que los principales recursos de la red que los jóvenes usuarios de medios han usado son: Wikipedia, Google y Youtube. Es en tal sentido, relevante construir procesos de capacitación a docentes y estudiantes que contribuyan a la indagación de fuentes educativas. Además, dentro de ello no se puede desvincular a este análisis los usos que hacen y partir desde allí las estrategias. A la consulta sobre cómo piensan los estudiantes que debería ser la educación en el siglo XXI, centran su atención a que debe fomentarse la tecnologización de la misma, propender espacios digitales antes que físicos para compartir tareas y aprendizajes además de aplicar estrategias desde la educación transmedia, empleando diferentes medios para articular la enseñanza y el aprendizaje en esta sociedad interconectada. Desde las instituciones educativas en una primera instancia se han iniciado procesos de alfabetización digital, que se enfocan en enseñar a manejar desde la visión artefactual el uso de las tecnologías digitales. Pero es relevante entonces en un segundo momento sobrepasar esta mirada instrumental, permitiendo a los jóvenes desde sus apropiaciones de la tecnología y el conocimiento crear espacios colaborativos y cooperativos hacia dotar de "habilidades para el procesamiento de datos e información hacia la construcción del conocimiento del aprendizaje" (Apolo, Bayés y Hermann, 2016, p. 235).

Por último, es importante mostrar que los estudios que analizan la producción más actualizada sobre redes sociales y su uso en las prácticas educativas indican, por una parte, que se trata de un tema que ha ocupado un lugar destacado en el ámbito académico internacional en los últimos años, dado el enorme impacto en la vida de las personas, y de otra parte, que es un campo emergente de investigación, aún muy centrado en estudios de naturaleza descriptiva, cuantitativa y con un mayor énfasis en el ámbito de la educación universitaria (Domínguez y López, 2015; Pérez et al.,2015; Vásquez y Cabrero, 2015; Rodríguez, Haya y Fernández, 2015). En ese sentido este trabajo contribuye a la consolidación de un campo de estudio que permitirá una mejor comprensión de un fenómeno que cambia y se complejiza al ritmo del crecimiento exponencial de los avances tecnológicos y sus impactos en la red.

\section{CONCLUSIONES}

A partir de los resultados presentados y con base en el análisis y discusión de estos se pueden enunciar las siguientes tres conclusiones principales:

1) los retos de la educación en la era digital se centran en repensar los aprendizajes en cuanto a educación fuera de espacios formales observando las oportunidades que brindan los procesos de aprendizajes no formales e informales;

2) a partir del contexto educativo estudiado se ha identificado que las tres redes sociales más utilizadas como son: Facebook, Instagram y Whatsapp; por tanto, es relevante aprovechar estos usos que se dan hacia entretenimiento y fortalecer estrategias que permitan su vinculación con fines educativos;

3) dentro de las perspectivas identificadas surge la relevancia de vincular el uso de las TIC al currículo de manera transversal generando procesos de alfabetización digital a todos los actores del proceso educativo desde perspectivas flexibles, abiertas y colaborativas.

\section{REFERENCIAS}

Abdelraheem, A.Y. y A. Ahmed, The Impact of Using Mobile Social Network Applications on Students' Social-Life, doi: 10.12973/iji.2018.1121a, International Journal of Instruction, 11(2), 1-14 (2018)

Akcaoglu, M. y N. Bowman, Using instructor-led Facebook groups to enhance students' perceptions of course content, doi 10.1016/j.chb.2016.05.029, Computers in Human Behavior, 65, 582-590 (2016)

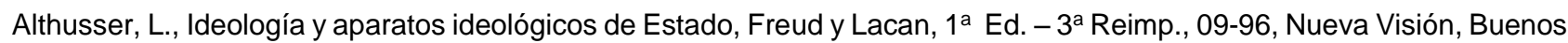
Aires, Argentina (2003)

Apolo, D., M. Bayés y A. Hermann, Cambios educativos en los procesos de lectura digital: la pedagogía del ciberespacio como estrategia de procesamiento de contenidos en la era de internet, doi: 10.15213, REDES.COM, Revista de Estudios para el Desarrollo Social de la Comunicación, (12), 222 (2016) 
Apolo, D., M. Rodríguez, A. Bueno y J. Solano, Uso de internet con fines educativos: aproximaciones hacia estudiantes universitarios en formación inicial docente; in Chem. El fin de un modelo de política by Herrero, J. y M. Trenta, (Eds.), pp. 1446-1460, Cuadernos Artesanos de comunicación, La Laguna, España (2017)

Caldevilla, D., Tipología, uso y consumo de las redes 2.0 en la sociedad digital actual, Documentación de las Ciencias de la Información, ISSN: 0210-4210, 33, 45-68 (2010)

Carrasco, J., Bodies go to school, a favor granted by our brains, doi: 10.4438/1988-592X-RE-2017-376-342, Revista de Educación, (376), 9-32 (2017)

Cobo, C. y J. Moravec, Aprendizaje invisible, Hacia una nueva ecología de la educación, pp. 09-191, Publicaciones y Ediciones de la Universidad de Barcelona, Barcelona, España (2011)

Colás, P., T. González y J. De Pablos, Juventud y redes sociales: Motivaciones y usos preferentes, doi: http://dx.doi.org/10.3916/C40-2013-02-01, Comunicar, (20)40, 15-23 (2013)

Domínguez, F., R. López, Uso de las redes sociales digitales entre los jóvenes universitarios en México. Hacia la construcción de un estado del conocimiento (2004-2014), Revista de Comunicación, ISSN: 1684-0933, 14, 48-69 (2015)

Fadol, Y., H. Aldamen y S. Saadullah, A comparative analysis of flipped, online and traditional teaching: A case of female Middle Eastern management students, doi.org/10.1016/j.jme.2018.04.003, The International Journal of Management Education, 16(2), 266-280 (2018)

Gómez, M., S. Roses y P. Farias, Descriptive Study of the Academic Use of Social Networks among University Students, doi: 10.3916/C38-2011-03-04, Comunicar, (38)20, 1-8 (2012)

Hermann, A., Prácticas educativas abiertas en entornos digitales: uso de las redes sociales en los Jóvenes Usuarios de Medios, Tesis de Magister, Subprograma de Investigación en Comunicación Digital en Educación, Universidad Nacional de Educación a Distancia, Madrid, España (2015)

INEC, Instituto Nacional de Estadística y Censos, Tecnologías de la Información TIC y Comunicaciones (TIC'S) (2016)

Kaun, A., M. Kyriakidou y J. Uldam, Political Agency at the Digital Crossroads? doi: 10.17645/mac.v4i4.690, Media and Communication, 4(4), 1-7 (2016)

Kristianto, B., Factors affecting social network use by students in Indonesia, doi.org/10.28945/3675, Journal of Information Technology Education, Research, 16, 69-103 (2017)

Lubua, E.W., A. Semlambo y P.D. Pretorius, Factors affecting the use of social media in the learning process, doi.org/10.4102/sajim.v19i1.764, South African Journal of Information Management, 19(1) a 764 (2017)

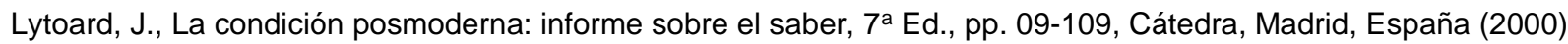

Martínez-Argüello, L., F. Hinojo-Lucena e I. Aznar-Díaz, Aplicación de las Tecnologías de la Información y la Comunicación (TIC) en los Procesos de Enseñanza- Aprendizaje por parte de los Profesores de Química, doi.org/10.4067/S07180764201800020004, Información Tecnológica, 29(2), 41-52 (2018)

Medina-Cruz, H., A. Lagunes-Domínguez, C. Torres-Gastelú, Percepciones de Estudiantes de Nivel Secundaria sobre el uso de las TIC en su Clase de Ciencias, doi.org/10.4067/S0718-07642018000400259, Información Tecnológica, 29(4), 259-266 (2018)

MINTEL, Ministerio de Telecomunicaciones y de la Sociedad de la Información, Observatorio TIC del Ecuador (2015)

Morillo, M., L. Sebastian y M. Casado, Design for regulated open access knowledge through internet, doi: 10.3989/arbor.2011.Extra-3n3141, Arbor-ciencia pensamiento y cultura, 187(3), 177-182 (2011)

Nehls, K. y J. Livengood, Facebook, crowdsourcing and the transition to college, doi.org/10.1080/0309877X.2017.1281885, Journal of Further and Higher Education, 42(3), 366-376 (2017)

Park, L., WeChat Red Bags: How International Students from China Use Social Media While Attending a Public University in California, UCLA. ProQuest ID: Park_ucla_0031N_14252. Merritt ID: ark:/13030/m5tt81mm (2016)

Parra, A., S. Gordo y S. D'Antonio. Social research applied to social networks. A methodological innovation for the analysis of Facebook Likes, DOI: 10.4185/RLCS-2014-1007en, Revista Latina de Comunicación Social, (69), 195- 212 (2014)

Pedro, L., C. Barbosa y C. Santos, A critical review of mobile learning integration in formal educational contexts, doi.org/10.1186/s41239-018-0091-4, International Journal of Educational Technology in Higher Education, 15(1), 1-10 (2018)

Pérez, M., M. Ortiz y M. Flores, Redes sociales en educación y propuestas metodológicas para su estudio. doi 14538571008, Ciencia, Docencia y Tecnología, 26 (50), 188-206 (2015)

Rodríguez, J., La práctica hace al maestro, in Chem. Cibercultura y prácticas de los profesores. Entre hermenéutica y educación, by D. Barragán, pp. 09-120, Ediciones Unisalle Bogotá, Colombia (2013)

Rodríguez-Hoyos, C., I. Haya, E. Fernández-Díaz, Research on SNS and education: The state of the art and its challenges, doi: 10.14742/ajet.995, Australasian Journal of Educational Technology, 31(1), 100-111 (2015)

Santamaría, F., Aprendizaje de espacios abiertos (2012)

Schei, V. e I. Nerbø, The invisible learning ceiling: Informal learning among preschool teachers and assistants in a Norwegian kindergarten, doi.org/10.1002/hrdq.21213, Human Resource Development Quarterly, 26(3), 299-328 (2015) 
Siibak, A., M. Forsman, P. Hernwall, Employing Creative Research Methods with Tweens in Estonia and Sweden: Reflections on a Case Study of Identity Construction on Social Networking Sites, doi: 10.1080/15228835.2012.745355, Journal of Technology in Human Services, 30 (3/4), 250-261 (2012)

Smith, S., (Re) Counting Meaningful Learning Experiences: Using Student-Created Reflective Videos to Make Invisible Learning Visible During PjBL Experiences, doi.org/10.7771/1541-5015.1541, Interdisciplinary Journal of Problem-Based Learning, 10(1), 1-16 (2016)

Toffler, A., La Tercera Ola, Bogotá, Plaza \& Janes (1980)

Vázquez, A., J. Cabero, Las redes sociales aplicadas a la formación, doi.org/10.5209/rev_RCED.2015.v26.47078, Revista Complutense de Educación, 26, 253-272 (2015)

Velásquez, A., G. Carrión y J. Yaguache, Use the Internet and Facebook in ecuadorian teenagers; Actas del 11th Iberian Conference on Information Systems and Technologies, 1-6, Islas Canarias, España 16-18 de Junio (2016) 
